\title{
Contralateral monoarthritis exacerbated chronic constriction injury-induced pain hypersensitivity through upregulating inducible nitric oxide synthase
}

This article was published in the following Dove Press journal: Journal of Pain Research

\section{Heng Zhao' \\ Shenghou Liu' \\ Chenhua Wang ${ }^{2}$ \\ Qingjie Wang ${ }^{3}$ \\ Wenguang Liu' \\ Mingzhi Gong'}

'Department of Orthopedics, The Second Hospital of Shandong University, Jinan, 250033,

Shandong, People's Republic of China; ${ }^{2}$ Department of Nuclear Medicine, The Second Hospital of Shandong University, Jinan, 250033, Shandong, People's Republic of

China; ${ }^{3}$ Department of Emergency, The Second Hospital of Shandong University, Jinan, 250033, Shandong, People's Republic of China
Correspondence: Mingzhi Gong Department of Orthopedics, The Second Hospital of Shandong University, Jinan, 250033, Shandong, People's Republic of China

Email gongmingzhi053।@I63.com
Introduction: High comorbidity of osteoarthritis (OA) and neuropathic pain has been reported in aged patients. Evidence shows that central sensitization of pain processing occurs in late-phase $\mathrm{OA}$ and may facilitate the development of neuropathic pain. Few studies reveal whether acute monoarthritis (MA) aggravates neuropathic pain on the opposite side of the body from the injury. Methods: To address whether neuropathic pain is affected by contralateral MA through distinct inflammatory pathway, MA was induced by intra-articular injection of complete Freund's adjuvant (CFA) into the right tibiotarsal joint, and neuropathic pain was established by chronic constriction injury (CCI) of the left sciatic nerve.

Results: We observed that MA aggravated mechanical allodynia and thermal hyperalgesia in CCI rats. Furthermore, MA affected the other side of the spinal cord in multiple aspects, including the upregulation of iNOS mRNA and the enhancement of forskolin-induced facilitation of excitatory synaptic transmission in the spinal cord dorsal horn substantia gelatinosa neurons. Discussion: Interestingly, intrathecal injection of $1400 \mathrm{~W}$, an antagonist of iNOS, attenuated intensity of pain behaviors in CCI rats with contralateral MA to similar levels in CCI rats without $\mathrm{MA}$, and also normalized the facilitatory effect of forskolin on excitatory synaptic transmission in the spinal cord dorsal horn neurons in contralateral MA rats. Therefore, contralateral MA worsened CCI-induced pain hypersensitivity probably through upregulating iNOS and enhancing the facilitation of synaptic transmission following CCI.

Conclusion: Inhibiting the iNOS might be a potential therapeutic strategy for concurrent OA and neuropathic pain.

Keywords: osteoarthritis, neuropathic pain, acute monoarthritis, chronic constriction injury

\section{Introduction}

Osteoarthritis (OA) is a common disease in aging population. ${ }^{1,2}$ As a pain disease, OA significantly impairs physical function and reduces quality of life. ${ }^{3}$ Neuropathic pain following a lesion/injury or disease of the somatosensory system ${ }^{4}$ is also very common in aging patients, ${ }^{5}$ especially in those with diabetes mellitus (diabetic neuropathy), herpes zoster (postherpetic neuralgia), low back pain (such as lumbar spinal stenosis), cancers, and stroke. ${ }^{6}$ Both OA and neuropathic pain show pain sensitization, ${ }^{6,7}$ and their comorbidity in aged patients is not rare. ${ }^{8}$ In patients with concurrent OA and neuropathic pain, the medication for either disease may not adequately relieve the symptoms. To improve the treatment of the two concurrent diseases, it is necessary to elucidate the pathophysiological relationship between these conditions. 
Chronic pain after arthritis is associated with chronic neuroinflammation in the peripheral or central nervous system. ${ }^{9,10}$ Levels of many proinflammatory cytokines (IL-1 $\beta$, TNF $\alpha$, IL-6, etc.) and chemokines (iNOS, eNOS, COX-2) are known to be increased in the primary afferent and dorsal horn neurons innervating arthritic joints. ${ }^{9}$ These elevated inflammatory mediators subsequently enhance synaptic transmission at the spinal cord level referred to as "central sensitization" and facilitate the development of neuropathic pain. ${ }^{11}$ Many studies dealing with OA use contralateral noninjured side as a control and focus on alterations in local or ipsilateral neuroinflammatory reactions. However, there is conflicting evidence, showing that inflammatory mediators in the contralateral side of arthritis rats are elevated in OA in some studies ${ }^{12-14}$ but remain unchanged in others. ${ }^{12,15,16}$ The discrepancy may come from the difference in animal models, the injection site of complete Freund's adjuvant (CFA), the concentration and dose of CFA, and the time line of the experiment. Furthermore, it is unclear about the roles of the inflammatory reactions following contralateral OA in the development of neuropathic pain.

The present study confirms that monoarthritis (MA) did affect the contralateral spinal cord and exacerbated pain hypersensitivity in chronic constriction injury (CCI). Additionally, the potential strategy that could reverse the exacerbation was also explored.

\section{Methods}

All animal care and experimental protocols follow the Guide for the Care and Use of Laboratory Animals (National Research Council, 1996, Washington, DC) and were approved by the Institute of Animal Care and Use Committee and the Office of Laboratory Animal Resources in the Second Hospital of Shandong University. Male Sprague Dawley rats (200-250 g) were used in this study. All rats were cage housed in an environment with a temperature of $22 \pm 2^{\circ} \mathrm{C}$, relative humidity between $45 \%$ and $75 \%$, and $12 \mathrm{~h}$ light-dark cycle. Water and food were provided ad libitum.

The rats were randomly divided into five groups: sham group (Sham, $\mathrm{n}=8$ ), received intra-articular injection of $50 \mu \mathrm{L}$ sterile saline; MA group $(n=8)$, received intra-articular injection of $50 \mu \mathrm{L} \mathrm{CFA}$; CCI group $(\mathrm{n}=8)$; and CCI + MA group $(n=16)$, having two subgroups: MA induction was performed either 2 days before $(n=8)$ or 2 days after $(n=8)$ CCI.

\section{Drug treatment}

Forskolin (Sigma-Aldrich Co., St Louis, MO, USA) was dissolved in dimethyl sulfoxide (DMSO) and applied to spinal cord slices in artificial cerebrospinal fluid (ACSF) at a final concentration of $20 \mu \mathrm{M} .1400 \mathrm{~W}$ (Selleck Chemicals, Houston, TX, USA) was dissolved in DMSO and intrathecally administrated to each animal (10 $\mu \mathrm{g}$ in $20 \mu \mathrm{L})$ right after CFA injection.

\section{Induction of MA}

MA was induced by injecting $50 \mu \mathrm{L}$ CFA (Sigma-Aldrich Co.) into the right tibiotarsal joint. This procedure was performed under isoflurane inhalation for anesthesia, as described previously. ${ }^{17}$ Briefly, the fossa of the lateral malleolus of the fibula was located, and a 28-gage needle was inserted from the gap between the tibiofibular bone and tarsus bone into the articular cavity. CFA $(50 \mu \mathrm{L})$ was injected into the articular cavity. The Sham rats were intra-articularly injected with sterile saline. The rats after CFA injection showed pronounced swelling in the right ankle measured using a plethysmometer (Ugo Basile, Milan, Italy). Animals without or with minimal swelling in the right ankle were discarded.

\section{Chronic constriction injury $(\mathrm{CCl})$}

CCI was performed as described previously. ${ }^{18,19}$ Briefly, a rat was anesthetized with intraperitoneal injection of sodium pentobarbital ( $40-60 \mathrm{mg} / \mathrm{kg}$ ); the sciatic nerve on the left side was exposed by making a skin incision and cutting through the connective tissue between the gluteus superficialis and biceps femoris muscles. Four chromic gut ligatures were tied loosely around the sciatic nerve with $1 \mathrm{~mm}$ intervals between two neighboring ligatures to just occlude but not arrest epineural blood flow. Muscle and skin layers were sutured separately. The rat was allowed to recover from surgery for $24 \mathrm{~h}$ before pain hypersensitivity tests began.

\section{Histology}

Hematoxylin-eosin (HE) staining was performed using $5 \mu \mathrm{m}$ sections of the right tibiotarsal joints of the Sham and MA rats. Briefly, the extremity of the right hindlimb containing tibiotarsal joints was dissociated and fixed in $10 \%$ neutral buffered formalin. For decalcification of tibia and tarsus, the specimen was immersed in $10 \%$ nitric acid and $1 \%$ ethylenediaminetetraacetic acid (EDTA) in PBS. Then, the sample was subjected to dehydration in a series of ethanol solutions with gradually increasing concentrations from $50 \%$ to $100 \%$. The dehydrated sample was embedded in paraffin and was sliced with a sliding microtome into $5 \mu \mathrm{m}$ thick sections, which were mounted onto glass slides. After dewaxing and washing, the sections were stained with $\mathrm{HE}$ and toluidine blue. The stained sections were examined using an upright microscope, equipped with a $4 \times$ objective. 
As illustrated in Figure S1, MA rats showed pathological damages in tibiotarsal joint, including deformation in tibia and tarsus; enhancement of mononuclear infiltration; and hyperplasia of synovial cells.

\section{Examination of pain behaviors}

Mechanical paw withdrawal threshold (PWT)

Mechanical PWT was calculated as described previously. ${ }^{20}$ Rats were individually placed in a box without bottom on an elevated metal mesh floor and were allowed to habituate for $\geq 30 \mathrm{~min}$ before examination. A series of calibrated von Frey hairs (Stoelting, Wood Dale, IL, USA) were applied one by one to the central region of the plantar surface of the left hind paw in an ascending order $(1.4,2,4,6,8,10,15$, and $26 \mathrm{~g}$ ). The 50\% PWT was determined using Dixon's up-down method.

\section{Thermal withdrawal latency}

Thermal withdrawal latency was measured using the thermal plantar analgesia instruments (IITC, Woodland Hills, CA, USA) as follows. Rats were placed on the glass floored testing cages for at least 30 min until the exploratory behavior ceased. The heat source was placed directly beneath the mid-plantar surface of the hind paw through a transparent glass surface. The delay of the hind paw withdrawal from the heat source, relative to the onset of thermal stimulus, was recorded. A cutoff time of $20 \mathrm{~s}$ was used to avoid tissue damage. The heat stimulation was repeated at least three times with an interstimulus interval of $5 \mathrm{~min}$, and then the mean of paw withdrawal latencies of individual rats was calculated.

\section{Quantitative polymerase chain reaction (qPCR)}

The left and right lumber segments (L3-L6) of the spinal cord from each rat were harvested separately $48 \mathrm{~h}$ after the injection of CFA for the induction of MA and immediately stored at $-80^{\circ} \mathrm{C}$. The samples were lysed in $500 \mu \mathrm{L}$ of TRIzol ${ }^{\circledR}$ reagent (Thermo Fisher Scientific, Waltham, MA, USA), and total
RNA was isolated according to the manufacturer's instruction. Integrity of RNA was confirmed in a denaturing agarose gel, and no contaminating DNA or RNA degradation was observed. Afterward, the RNA was reverse transcribed using $2 \mu \mathrm{g}$ total RNA as the template using a reverse transcription polymerase chain reaction (RT-PCR) kit (Promega Corporation, Fitchburg, WI, USA). Reverse transcription was performed at $45^{\circ} \mathrm{C}$ for $45 \mathrm{~min}$, followed by $5 \mathrm{~min}$ heating at $92^{\circ} \mathrm{C}$. The real-time PCR was performed in Roche LightCycler 480 (Hoffman-La Roche Ltd., Basel, Switzerland) with Maxima SYBR Green/ROX qPCR Master Mix (2×) (Thermo Fisher Scientific) for detection. Samples were amplified in $25 \mu \mathrm{L}$ reaction mixtures, containing $0.3 \mu \mathrm{M}$ forward and reverse primers, $12.5 \mu \mathrm{L}$ Maxima SYBR Green/ROX qPCR Master Mix (2×), template DNA $\leq 500 \mathrm{ng} /$ reaction, and nuclease-free water. All primers were designed using Primer-BLAST design software (NIH, Bethesda, MD, USA) and were synthesized by Sangon Biotech (Shanghai, People's Republic of China). The primers we used are listed in Table 1. All PCRs were performed with the following parameters: $10 \mathrm{~min}$ at $95^{\circ} \mathrm{C}$ for initial denaturation, 35 cycles of sequential steps $\left(15 \mathrm{~s}\right.$ at $95^{\circ} \mathrm{C}$ for denaturation, $1 \mathrm{~min}$ at $55^{\circ} \mathrm{C}-64^{\circ} \mathrm{C}$ for annealing, $30 \mathrm{~s}$ at $72^{\circ} \mathrm{C}$ for extension), and then a $7 \mathrm{~min}$ final incubation at $72^{\circ} \mathrm{C}$. Each sample was tested in triplicates. The relative expression was calculated using the $\Delta \Delta C_{q}$ method $^{21}$ and optimized with a standard curve to confirm the high specificity.

\section{Patch-clamp recording}

Whole-cell patch-clamp recordings were made from substantia gelatinosa (SG) neurons in lumbar spinal cord slices as described previously. ${ }^{22}$

In brief, $48 \mathrm{~h}$ after the injection of CFA, the rats were suffocated to death with $\mathrm{CO}_{2}$ and were decapitated. After laminectomy, the lumber spinal cord was removed and sliced $(300 \mu \mathrm{m})$ with a vibratome (VT-1200S; Leica Inc., Wetzlar, Germany) in ice-cold-modified, sucrose-based artificial cerebral spinal fluid (SACSF) saturated with $95 \% \mathrm{O}_{2} / 5 \% \mathrm{CO}_{2}$ containing (mM) 250 sucrose, $2.5 \mathrm{KCl}, 1.2 \mathrm{NaH}_{2} \mathrm{PO}_{4}, 1.2$

Table I Sequences of primers used in RT-PCR

\begin{tabular}{llll}
\hline Gene & Forward 5'-3' & Reverse $\mathbf{5}^{\prime} \mathbf{- 3}$ & Annealing temperature ('C) \\
\hline IL-I $\beta$ & CATCTTTGAAGAAGAGCCCG & AACTATGTCCCGACCATTGC & 60 \\
INF- $\gamma$ & AGGAAAGAGCCTCCTCTTGG & TCTACCCCAGAATCAGCACC & 57 \\
IL-I7A & CTGGACTCTGAGCCGCAA & TGCCTCCCAGATCACAGAAG & 6 I \\
TNF- $\alpha$ & CGGGGTGATCGGTCCCAACAA & GTGGTTTGCTACGACGTGGGC & 60 \\
COX-2 & TGTATGCTACCATCTGGCTTCGG & GTTTGGAACAGTCGCTCGTCATC & 60 \\
iNOS & CCTTGTTCAGCTACGCCTTC & CATGGTGAACACGTTCTTGG & 55 \\
GAPDH & TGCACCACCAACTGCTTAGC & GGCATGGACTGTGGTCATGAG & 60 \\
\hline
\end{tabular}

Abbreviation: RT-PCR, reverse transcription polymerase chain reaction. 
$\mathrm{MgCl}_{2}, 2.4 \mathrm{CaCl}_{2}, 26 \mathrm{NaHCO}_{3}$, and 11 glucose. Slices were first recovered at $32^{\circ} \mathrm{C}$ for at least $1 \mathrm{~h}$ in SACSF and then kept at room temperature $\left(22-24^{\circ} \mathrm{C}\right)$ in ACSF bubbled with $95 \% \mathrm{O}_{2} / 5 \% \mathrm{CO}_{2}$ containing $(\mathrm{mM}) 126 \mathrm{NaCl}, 2.5 \mathrm{KCl}, 1.2$ $\mathrm{NaH}_{2} \mathrm{PO}_{4}, 1.2 \mathrm{MgCl}_{2}, 2.4 \mathrm{CaCl}_{2}, 26 \mathrm{NaHCO}_{3}$, and 11 glucose for at least $30 \mathrm{~min}$. Then, a slice was placed in a recording chamber and perfused (1.5-2.0 $\mathrm{mL} / \mathrm{min})$ with ACSF, which was constantly bubbled with $95 \% \mathrm{O}_{2} / 5 \% \mathrm{CO}_{2}$ continually. In all, $1 \mu \mathrm{M}$ tetrodotoxin (TTX) and $10 \mu \mathrm{M}$ bicuculline were applied continually with perfusate when miniature excitatory postsynaptic currents (mEPSCs) were recorded. Drugs were applied with the perfusate as needed.

The neurons in spinal cord slices were visualized with an upright microscope (Eclipse FN1; Nikon Instruments, Melville, NY, USA) and near-infrared illumination. Whole-cell patch-clamp techniques were used to record electrophysiological signals with a MultiClamp 700B amplifier (Axon Instruments, Molecular Devices LLC, Sunnyvale, CA, USA), Digidata 1440 analog-to-digital converters (Axon Instruments), and pClamp 10.2 software (Axon Instruments). Data were sampled at $10 \mathrm{kHz}$ and filtered at $2 \mathrm{kHz}$. The patch electrode had a resistance of 5-7 $\mathrm{M} \Omega$ when filled with pipette solution containing (mM) 135 potassium gluconate, $5 \mathrm{KCl}, 5$ ethylene glycol-bis( $\beta$-aminoethyl ether)-N,N,N',N'-tetraacetic acid (EGTA), $0.5 \mathrm{CaCl}_{2}, 10 \mathrm{HEPES}, 2 \mathrm{Mg}$-ATP, and $0.1 \mathrm{GTP}$. The $\mathrm{pH}$ of these solutions was adjusted to 7.2 with Tris-base, and the osmolarity was adjusted to $300 \mathrm{mOsm}$ with sucrose. The junction potential between the patch pipette and bath solution was nulled before gigaseal formation. Series resistance was monitored without compensation throughout the experiment. All recordings were done at a temperature of $32 \pm 0.5^{\circ} \mathrm{C}$.

mEPSCs were counted and analyzed using Clampfit 10.3 (Molecular Devices LLC) after the traces were low-pass filtered at $2 \mathrm{kHz}$. Waveform templates for mEPSCs were defined according to the rise and decay times. The events that match the waveform and exceed the amplitude threshold were counted. The frequency and amplitude of mEPSCs before and after drug application were compared.

\section{Statistical analysis}

Data are presented as mean \pm standard error of measurement (SEM). Statistical significance was determined using parametric or nonparametric one-way or two-way analysis of variance (ANOVA) with Bonferroni test for pairwise comparison, using GraphPad Prism ${ }^{\circledR} 5$ software (GraphPad Software, Inc., La Jolla, CA, USA). Difference was considered as statistically significant when $p$ value was $<0.05$.

\section{Results Effect of contralateral MA on CCl- induced hypersensitivity to pain}

First, we tested whether CCI-induced ipsilateral nociceptive hypersensitivity in rats can be facilitated by contralateral MA. In this set of experiments, we established contralateral MA 2 days before or after CCI to clarify whether MA affects the development or maintenance of CCI-induced hypersensitivity to pain.

In our observation, there was no significant difference in baseline mechanical PWT and thermal paw withdrawal latency among the groups that received different treatments (Figures $1 \mathrm{~A}$ and $\mathrm{B}$ and $2 \mathrm{~A}$ and $\mathrm{B}$ ). In comparison with the sham group, CCI groups showed decreased mechanical PWT (Figures 1A and 2A) and thermal paw withdrawal latency on the ipsilateral side (Figures $1 \mathrm{~B}$ and $2 \mathrm{~B}$ ) that were maintained up to 3 weeks, confirming the successful establishment of CCI-neuropathic pain models, displaying mechanical allodynia and thermal hyperalgesia.

Interestingly, although MA alone did not affect pain sensation of the paw on the other side (Figure S2), contralateral MA performed 2 days after CCI worsened CCI-induced mechanical allodynia in 2 days (Figure 1A) and thermal hyperalgesia in 4 days (Figure 1B), and the deterioration of pain hypersensitivity persisted up to 3 weeks. Similarly, applying contralateral MA 2 days before CCI exacerbated CCI-induced ipsilateral pain hypersensitivity, and the deterioration remained up to 3 weeks after $\mathrm{CCI}$ (Figure 2A and $\mathrm{B}$ ). Note that contralateral MA before CCI reduced mechanical PWT immediately after CCI and shortened thermal paw withdrawal latency 6 days after CCI.

Taken together, our data suggest that contralateral MA before and after CCI both exacerbated CCI-induced mechanical allodynia and thermal hyperalgesia.

\section{mRNA levels of inflammatory mediators in both sides of the spinal cord after MA}

We examined mRNA levels of cytokines and chemokines in the lumber spinal cord ipsilateral and contralateral to the side that MA was induced. We observed that MA significantly increased mRNA levels of IL-1 $\beta$, INF- $\gamma$, IL-17A, TNF $\alpha$, and COX-2 in the ipsilateral but not in the contralateral lumber spinal cord of MA rats compared to Sham rats (Figure 3A-E). Interestingly, mRNA levels of iNOS in MA rats were increased bilaterally in the spinal cord in comparison with those of Sham rats. The levels of iNOS mRNA increased by sevenfold in the ipsilateral spinal cord and by 

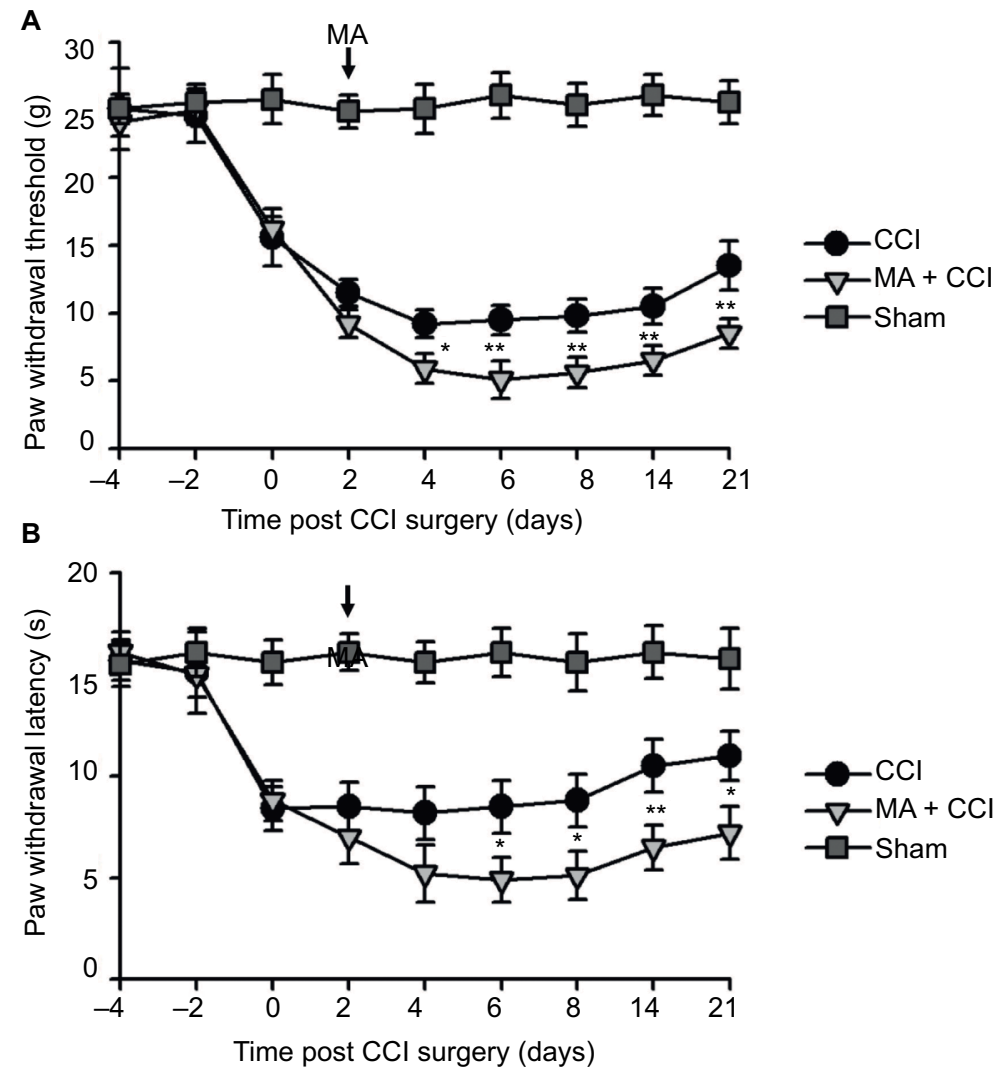

Figure I Contralateral MA induced 2 days after $\mathrm{CCl}$ exacerbated pain behaviors.

Notes: $\mathrm{CCl}$ was performed in the left sciatic nerve to establish neuropathic pain in rat models, and CFA was injected in the right tibiotarsal joint to induce MA. In the sham group, rats were injected in the right tibiotarsal joint with saline instead of CFA. The von Frey filament tests and the thermal plantar tests were respectively applied to assess mechanical allodynia (A) and heat hyperalgesia (B) in Sham, $\mathrm{CCl}$, and $\mathrm{CCl}+\mathrm{MA}$ rats. Data are shown as mean $\pm \mathrm{SEM}$. $\mathrm{n}=7$ in each group. Statistical significance of the difference between sham, $\mathrm{CCl}$, and $\mathrm{MA}+\mathrm{CCl}$ rats at each time point was analyzed with two-way ANOVA and Bonferroni test for pairwise comparison. $\mathrm{CCl}$ vs $\mathrm{CCl}+$ MA: $* p<0.05, * * p<0.01$, and $* * * p<0.001$.

Abbreviations: ANOVA, analysis of variance; $\mathrm{CCl}$, chronic constriction injury; CFA, complete Freund's adjuvant; MA, monoarthritis; SEM, standard error of measurement.

sixfold in the contralateral side (Figure 3F). There was no statistically significant difference between two sides. Thus, following MA, elevation in iNOS mRNA levels was similar in two sides of the spinal cords.

\section{iNOS enhances forskolin-induced facilitation of synaptic transmission}

Since increased levels of iNOS in the spinal cord dorsal horn have been reported to be associated with neuronal excitability and long-term potentiation, ${ }^{23,24}$ we next studied whether MAinduced upregulation of iNOS affects synaptic transmission in the opposite side of the spinal cord.

First, mEPSCs were recorded using the whole-cell patch-clamp technique from the SG neurons in one side of spinal cord opposite to the side sham or MA operation was performed. The frequency and amplitude of mEPSCs were at the same levels in Sham and MA rats (Figure 4A). Forskolin
(50 $\mu \mathrm{M}, 5 \mathrm{~min}$ ), an activator of adenylyl cyclase, when bath-applied with perfusate, induced a sustained increase in mEPSC frequency and amplitude in both Sham and MA rats, and the effects reached maximal levels 20 min after the initiation of forskolin perfusion. Interestingly, the enhancement of mEPSCs was stronger in MA rats than in Sham rats (Figure 4B and C). The results suggest that synaptic facilitation by forskolin is significantly greater in contralateral spinal cord SG neurons of MA rats.

As we found that MA led to elevated iNOS mRNA in the contralateral side of the spinal cord, we tested whether blocking iNOS eliminates the enhanced effects of forskolin on mEPSCs in MA rats. To address this question, we intrathecally administered $1400 \mathrm{~W}(10 \mu \mathrm{g})$, an iNOS antagonist, right after intra-articular injection of CFA. In MA rats, 1400W pretreatment attenuated forskolin-induced enhancement of mEPSC frequency and amplitude in contralateral SG neurons 


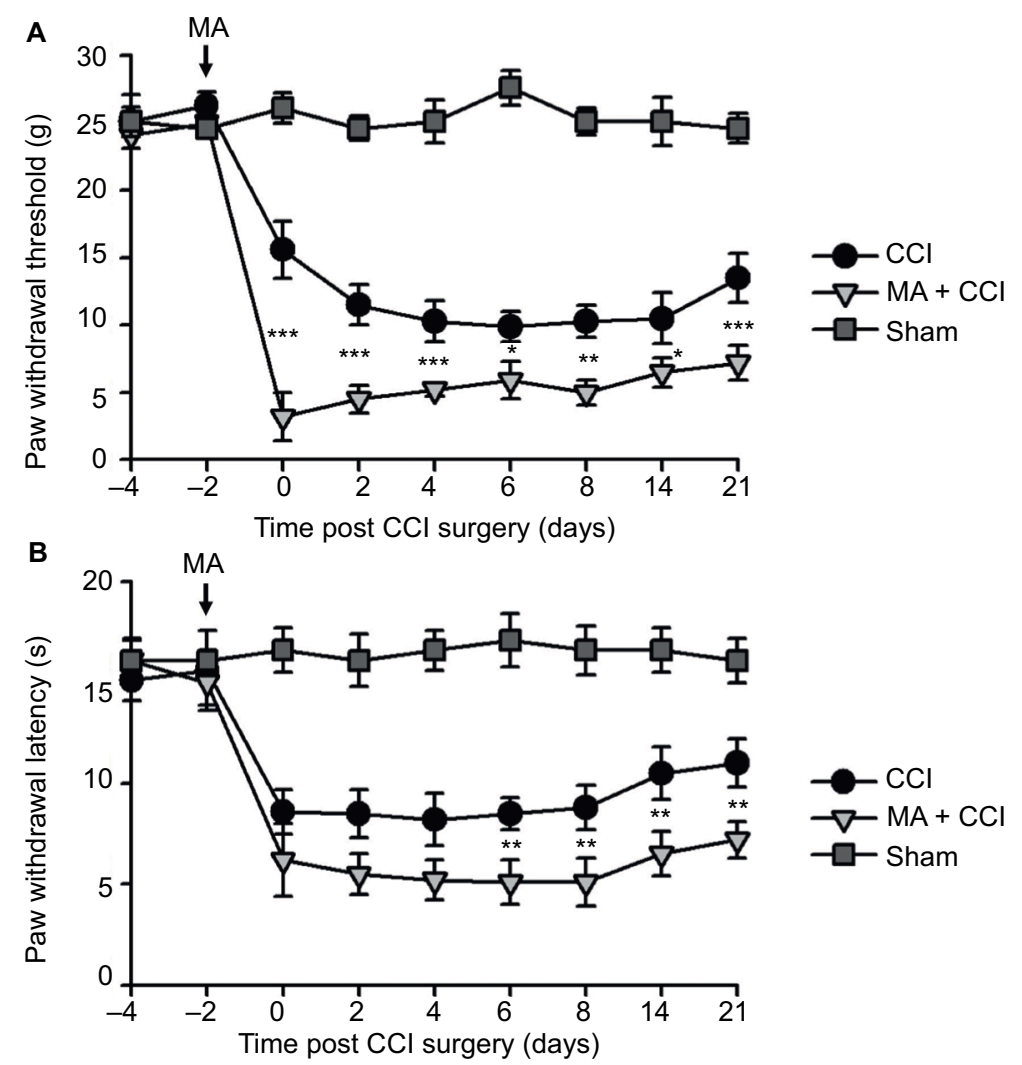

Figure 2 Contralateral MA 2 days before $\mathrm{CCl}$ exacerbated pain behaviors in $\mathrm{CCl}$ rats.

Notes: $\mathrm{CCl}$ was performed in the left sciatic nerve, and CFA was injected in the right tibiotarsal joint 2 days before $\mathrm{CCl}$. In the Sham group, rats were injected with saline instead of CFA. Right-side MA enhanced $\mathrm{CCl}$-induced mechanical allodynia (A) and heat hyperalgesia (B) in the left hind paw. Data are shown as mean \pm SEM. $n=7$ in each group. Statistical significance of the difference at each time point among Sham, $\mathrm{CCl}$, and $\mathrm{MA}+\mathrm{CCl}$ groups were determined with two-way ANOVA and Bonferroni test for pairwise comparison. $\mathrm{CCl}$ vs $\mathrm{CCl}+\mathrm{MA}:{ }^{*} p<0.05$, ${ }^{* *} p<0.01$, and ${ }^{* * *} p<0.001$.

Abbreviations: ANOVA, analysis of variance; $\mathrm{CCl}$, chronic constriction injury; CFA, complete Freund's adjuvant; MA, monoarthritis; SEM, standard error of measurement.

to the level similar to Sham rats (Figure 4A-C). These data suggest that upregulation of iNOS in the contralateral side of the spinal cord in MA rats contributes to enhanced forskolininduced facilitation of synaptic transmission.

\section{Effect of I400W on MA-sensitized pain in contralateral $\mathrm{CCl}$ models}

We observed that MA led to multiple levels of alterations in the contralateral side of the spinal cord, including elevated expression of iNOS and worsened pain hypersensitivity. We were wondering whether elevated iNOS expression could contribute to pain hypersensitivity. To answer this question, we tested the effect of $1400 \mathrm{~W}$ on exacerbation of contralateral CCI-induced pain hypersensitivity following MA. As showed in Figure 5A and B, in comparison with Sham rats, all rats in CCI, MA + CCI, and MA + CCI + 1400W groups developed mechanical allodynia and thermal hyperalgesia, which remained stable throughout the experimental period.
Additionally, $1400 \mathrm{~W}$ pretreatment significantly attenuated mechanical allodynia and thermal hyperalgesia in the MA $+\mathrm{CCI}$ group to a level comparable to the CCI group. These data suggest that inhibition of iNOS can reverse MA-induced deterioration of contralateral neuropathic pain.

\section{Discussion}

The aim of this study is to evaluate the effect of contralateral MA on CCI-induced neuropathic pain. We observed that in CCI models, pre- or post-induction of contralateral MA enhanced CCI-induced mechanical allodynia and thermal hyperalgesia. We also found that contralateral MA similarly elevated the levels of iNOS mRNA in both sides of the spinal cord. Although MA had no effect on synaptic transmission on the opposite side, it enhanced forskolin-induced facilitation of synaptic transmission, and this enhancement was eliminated by intrathecal administration of iNOS inhibitor. Furthermore, contralateral MA-induced aggravation of 
A

IL-2 $\beta$
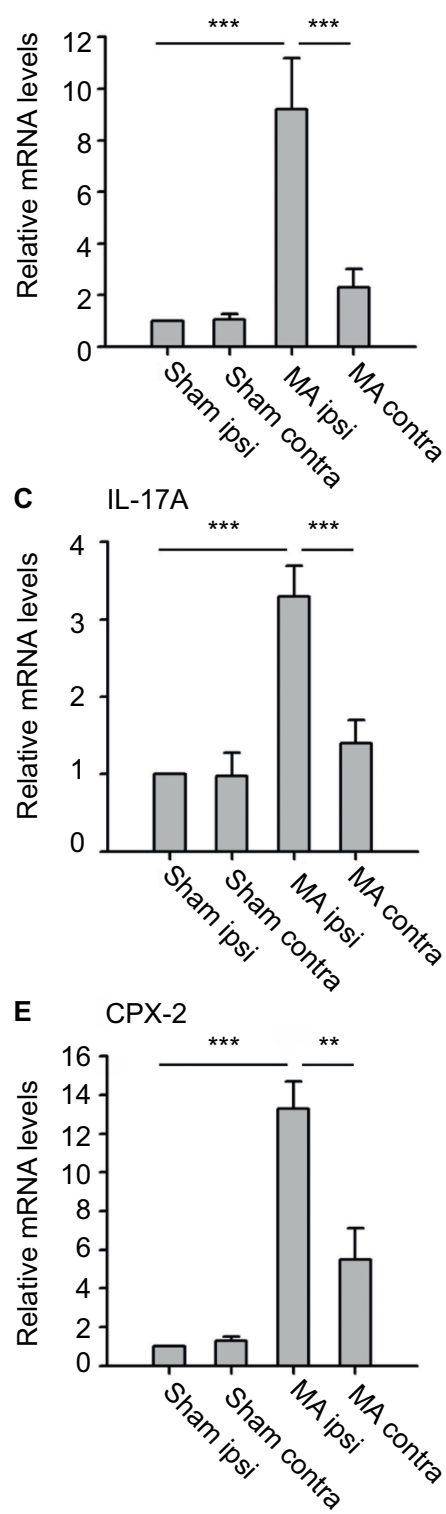

B INF- $\gamma$

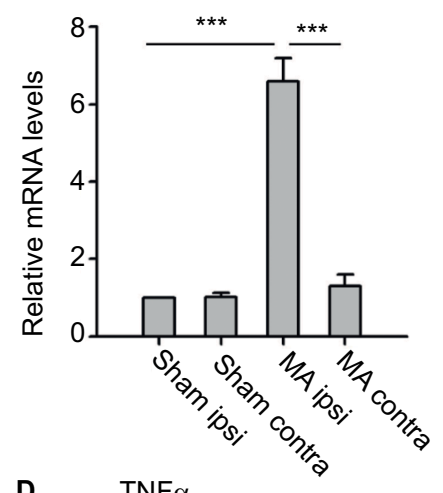

D $\quad \mathrm{TNF} \alpha$

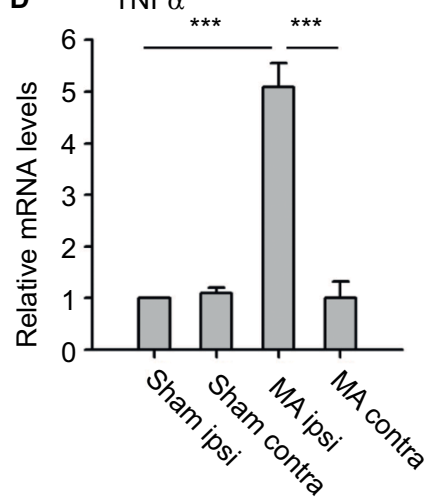

$\mathbf{F}$

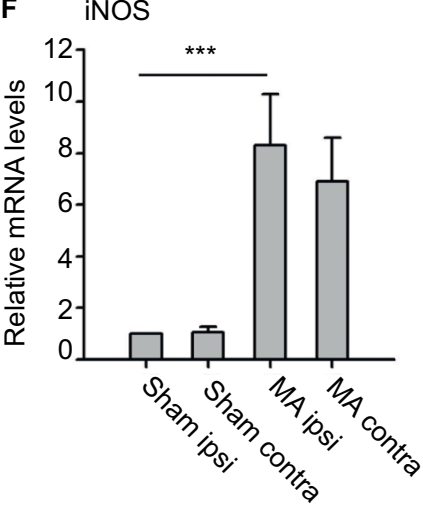

Figure 3 Effects of MA on mRNA levels of several inflammatory mediators in either side of the lumbar segments of the spinal cord.

Notes: qPCR was used to measure mRNA levels of IL-I $\beta$ (A), INF- $\gamma(\mathbf{B})$, IL-I7A (C), TNF- $\alpha(\mathbf{D})$, COX-2 (E), and iNOS (F) in the ipsilateral and contralateral spinal cord tissues of MA and Sham rats. Data are shown as mean \pm SEM. $n=7$ in each group. Statistical significance of difference was determined using one-way ANOVA on ranks and Dunn's test for pairwise comparison. $* * 0<0.01$ and $* * * p<0.001$.

Abbreviations: ANOVA, analysis of variance; contra, contralateral; ipsi, ipsilateral; MA, monoarthritis; qPCR, quantitative polymerase chain reaction; SEM, standard error of measurement.

mechanical allodynia and thermal hyperalgesia in CCI was attenuated by $1400 \mathrm{~W}$ pretreatment to the similar levels in CCI rats without MA. Overall, upregulation of iNOS mRNA following contralateral MA might be a mechanism underlying exacerbation of pain hypersensitivity in neuropathic pain.

Although peripheral nerve injury is directly associated with neuronal dysfunction (hyperexcitability) in spinal cord dorsal horn, inflammatory changes play critical roles in worsening the neuronal dysfunction. ${ }^{25}$ Especially, widespread inflammatory reaction usually exceeds the borders of tissue damage. Our study specifically evaluated mRNA levels of several proinflammatory cytokines and chemokines in the lumber spinal cord following MA. Although the upregulation of IL- $1 \beta$, INF- $\gamma$, IL-17A, TNF- $\alpha$, and COX-2 in the spinal cord remained restricted on the side of MA, iNOS mRNA in the spinal cord was bilaterally elevated by MA. These results suggest that inflammatory mediators in the spinal cord were differentially regulated by MA with diverse patterns.

It is noteworthy that the magnitudes of iNOS upregulation on both sides were comparable. Increased expression of 
A

Basal

Sham
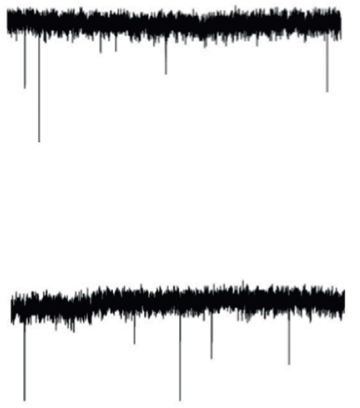

MA
MA+
1400W
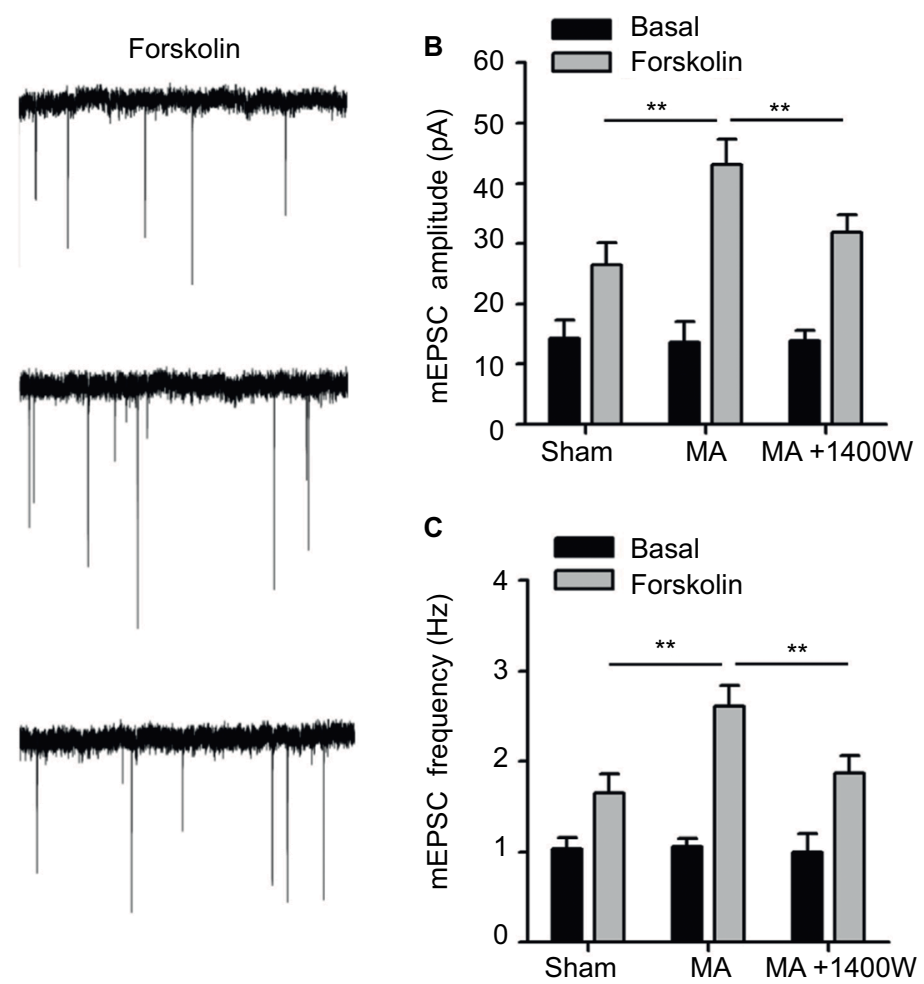

Figure 4 Effects of MA on excitatory synaptic transmission in the contralateral side of the spinal cord.

Notes: MA was induced by injecting CFA in the right tibiotarsal joint, and in some rats, I400W (10 $\mu \mathrm{g})$ was intrathecally injected right after CFA injection. mEPSCs were recorded using the patch-clamp technique $\left(V_{H}=-65 \mathrm{mV}\right)$ from lamina II SG neurons in the contralateral side of spinal cord in the presence of $10 \mu \mathrm{M}$ bicuculline and I $\mu \mathrm{M}$ TTX. (A) Representative traces of mEPSCs before and $20 \mathrm{~min}$ after forskolin perfusion $(50 \mu \mathrm{M}, 5 \mathrm{~min})$. Statistical results of the amplitude (B) and frequency (C) of $\mathrm{mEPSCs}$. Data are shown as mean \pm SEM. $n=10-15$ in each group. Statistical significance of difference was assessed using one-way ANOVA on ranks and Dunn's test for pairwise comparison. ${ }^{* *} p<0.01$.

Abbreviations: ANOVA, analysis of variance; CFA, complete Freund's adjuvant; MA, monoarthritis; mEPSC, miniature excitatory postsynaptic current; SEM, standard error of measurement; SG, substantia gelatinosa; TTX, tetrodotoxin.

contralateral iNOS has been shown in the lumbar spinal cord of arthritis rats by other research groups. ${ }^{14,16,26}$ The differences in iNOS expression levels found in these studies could result from diverse paradigms for pain induction and/or the timing and methods for iNOS examination.

The iNOS is not constitutively expressed but must be induced to be synthesized and become abundant in a variety of cell types, including neurons and glial cells. ${ }^{27}$ Previous studies demonstrated that iNOS is involved in the development of inflammatory neuropathic pain. ${ }^{14,28}$ The expression of iNOS in lamina II neurons has been shown to contribute to spinal nociceptive processing in several pain models. ${ }^{24,29-31}$ However, glial activation could account, at least in part, for the observed increases in iNOS levels in the spinal cord dorsal horn following MA. Investigations are warranted to determine to what extent the increased iNOS depends on the altered neuronal and/or glial function in the spinal cord dorsal horn.

It is noteworthy that, in addition to MA-induced iNOS, 1400W may directly affect neuropathic pain. A previous study has reported that $1400 \mathrm{~W}$ might exert its analgesic effects by reducing iNOS and altering the balance between the proinflammatory (IL-1 $\alpha$ and IL-1 $\beta$ ) and anti-inflammatory (IL-10) cytokines. ${ }^{32}$ In our study, $1400 \mathrm{~W}$ partly reversed the pain behavior from the animal receiving MA + CCI. It is possible that part of this rescue effect can be attributed to the ameliorated CCI pain. In order to directly test the role of MA-induced iNOS in the facilitation of pain development, future study needs to specifically and precisely inhibit contralateral iNOS expression induced by MA, e.g., viral vector-mediated knockdown of iNOS mRNA. Facilitation of excitatory synaptic transmission in the spinal cord dorsal horn is thought to contribute to persistence of clinical pain and is fundamental for the central nervous system to generate pain hypersensitivity. ${ }^{22}$ Few electrophysiological studies have been carried out to directly study the functional plasticity that occurs in contralateral spinal cord following the induction of MA. We showed here that excitatory synapses on contralateral SG neurons of MA rats had normal baseline activity, consistent with our data (Figure S2) and a previous 

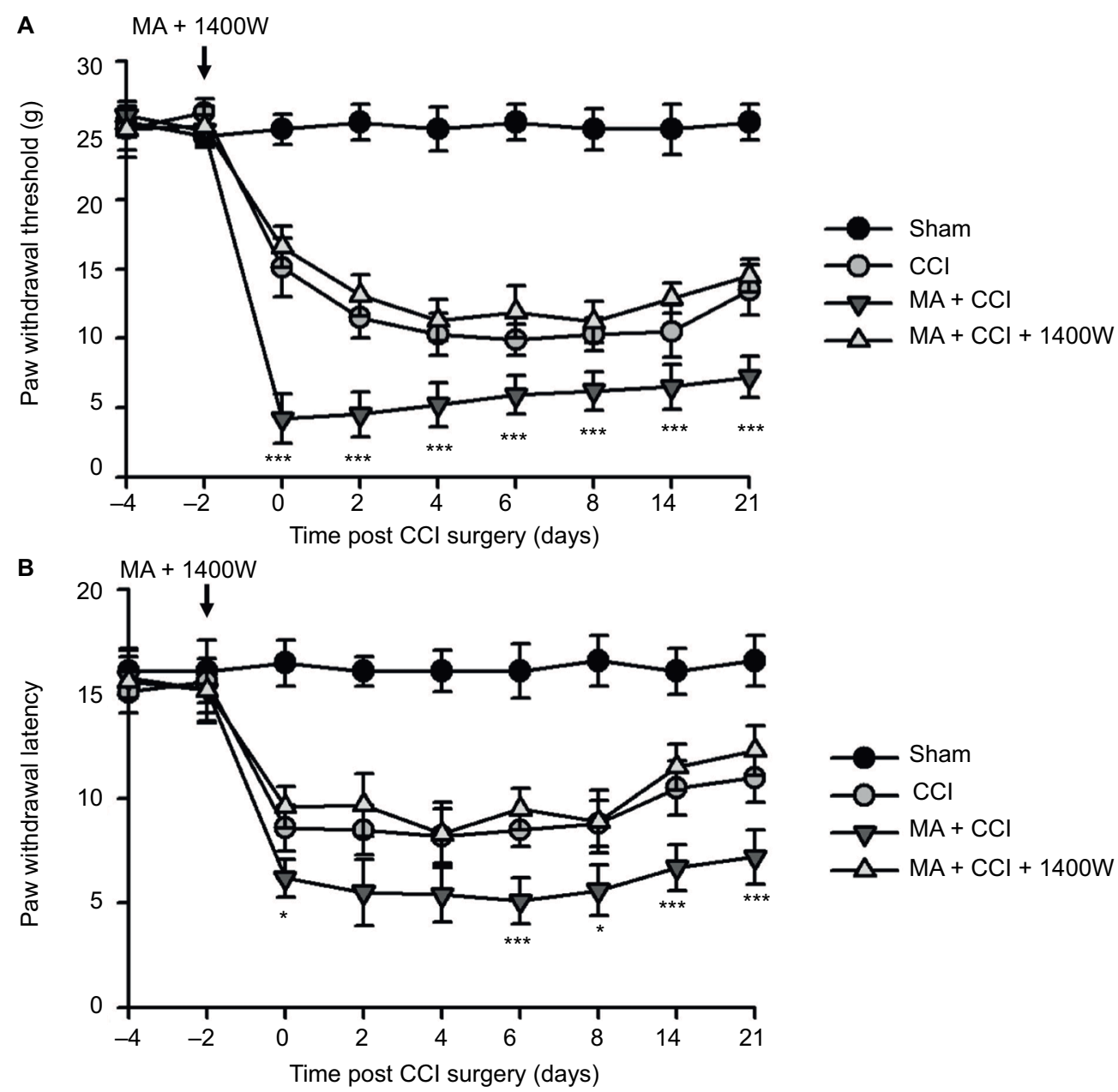

Figure 5 Effects of iNOS inhibition on MA facilitation of contralateral neuropathic pain.

Notes: Two days before $\mathrm{CCl}$ surgery, I400W (I0 $\mu \mathrm{g})$ was intrathecally injected immediately after CFA injection in the right tibiotarsal joint. CCl-induced mechanical allodynia (A) and heat hyperalgesia (B). Sham rats were injected with saline instead of CFA. Data are shown as mean \pm SEM. $n=9$ in each group. Statistical significance of difference at each time point among groups was assessed using two-way ANOVA and Bonferroni test for pairwise comparison. MA + CCl vs $\mathrm{MA}+\mathrm{CCl}+1400 \mathrm{~W}$ : $* 0<0.05$ and $* * * p<0.001$.

Abbreviations: ANOVA, analysis of variance; $\mathrm{CCl}$, chronic constriction injury; CFA, complete Freund's adjuvant; MA, monoarthritis; SEM, standard error of measurement.

study, showing that unilateral arthritis rats did not show pain hypersensitivity in the contralateral side. ${ }^{17}$ We argue that in our experimental condition, MA-induced upregulation of iNOS in the contralateral dorsal horn, by itself, may not be enough to enhance excitatory synaptic transmission and induce hyperalgesia. As activation of cyclic adenosine monophosphate (cAMP) response element binding protein (CREB), a transcription factor, parallels with the appearance and maintenance of pain hypersensitivity in CCI rats, ${ }^{33}$ to mimic this pathophysiological condition, we tested the effect of forskolin on mEPSCs, because, as an agonist of adenosine monophosphate (AMP) cyclase, forskolin leads to accumulation of cAMP, which binds CREB and triggers its activation. Intriguingly, stronger forskolin-induced potentiation of excitatory synaptic transmission in the spinal cord was observed in the side contralateral to MA (Figure 4).
Interestingly, this facilitation of synaptic transmission was abolished by a specific iNOS blocker, 1400W (Figure 4), suggesting an involvement of NO-cGMP cascade, downstream of iNOS activation.

Our behavioral data show that mechanical allodynia and thermal hyperalgesia in CCI rats were strengthened by contralateral MA. This phenomenon occurred 2 days after MA induction and persisted up to 3 weeks, indicating that MA can induce rapid and persistent changes in the contralateral side of the spinal cord. Upregulation of iNOS induced by contralateral MA possibly participated in the persistently enhanced nociceptive responses, because intrathecal injection of $1400 \mathrm{~W}$ attenuated pain behaviors in CCI rats with MA to the levels similar to those in CCI rats without MA. These data suggest that contralateral MA-induced potentiation of pain behaviors in the CCI model possibly depends on the 
elevated expression of iNOS. This notion was supported by previous reports showing that an increased expression of iNOS in the spinal cord dorsal horn was associated with neuron excitability and long-term potentiation that contribute to central hyperalgesia. ${ }^{23,24,34}$

Taken together, we provide evidence to support that an initially localized arthritis is able to elevate the levels of iNOS in the contralateral side of the spinal cord. This may in turn mediate facilitation of central sensitization (hyperactivity in excitatory synaptic transmission), and exacerbate symptoms of neuropathic pain. This study suggests that patients with neuropathic pain may have higher intensity of pain when they have comorbid OA. We provide evidence for the rationale that iNOS inhibitors might have therapeutic potential in the treatment of neuropathic pain comorbid with arthritis.

\section{Acknowledgment}

This study was supported by the Key Research and Development Plan of Shandong Province (number 2015GSF118107).

\section{Disclosure}

The authors report no conflicts of interest in this work.

\section{References}

1. Neogi T. The epidemiology and impact of pain in osteoarthritis. Osteoarthritis Cartilage. 2013;21(9):1145-1153.

2. Felson DT, Naimark A, Anderson J, Kazis L, Castelli W, Meenan RF. The prevalence of knee osteoarthritis in the elderly. The Framingham osteoarthritis study. Arthritis Rheum. 1987;30(8):914-918.

3. Salaffi F, Carotti M, Stancati A, Grassi W. Health-related quality of life in older adults with symptomatic hip and knee osteoarthritis: a comparison with matched healthy controls. Aging Clin Exp Res. 2005;17(4):255-263.

4. Ochoa JL. Neuropathic pain: redefinition and a grading system for clinical and research purposes. Neurology. 2009;72(14):1282-1283.

5. Haslam C, Nurmikko T. Pharmacological treatment of neuropathic pain in older persons. Clin Interv Aging. 2008;3(1):111-120.

6. Colloca L, Ludman T, Bouhassira D, et al. Neuropathic pain. Nat Rev Dis Primers. 2017;3:17002.

7. Fingleton C, Smart K, Moloney N, Fullen BM, Doody C. Pain sensitization in people with knee osteoarthritis: a systematic review and meta-analysis. Osteoarthritis Cartilage. 2015;23(7):1043-1056.

8. Power JD, Perruccio AV, Gandhi R, et al. Neuropathic pain in end-stage hip and knee osteoarthritis: differential associations with patient-reported pain at rest and pain on activity. Osteoarthritis Cartilage. 2018;26(3):363-369.

9. Lee AS, Ellman MB, Yan D, et al. A current review of molecular mechanisms regarding osteoarthritis and pain. Gene. 2013;527(2):440-447.

10. Miller RE, Miller RJ, Malfait AM. Osteoarthritis joint pain: the cytokine connection. Cytokine. 2014;70(2):185-193.

11. Lluch E, Torres R, Nijs J, Van Oosterwijck J. Evidence for central sensitization in patients with osteoarthritis pain: a systematic literature review. Eur J Pain. 2014;18(10):1367-1375.

12. Donaldson LF, Seckl JR, McQueen DS. A discrete adjuvant-induced monoarthritis in the rat: effects of adjuvant dose. J Neurosci Methods. 1993;49(1-2):5-10.

13. Beiche F, Scheuerer S, Brune K, Geisslinger G, Goppelt-Struebe M. Up-regulation of cyclooxygenase-2 mRNA in the rat spinal cord following peripheral inflammation. FEBS Lett. 1996;390(2):165-169.
14. Dolan S, Field LC, Nolan AM. The role of nitric oxide and prostaglandin signaling pathways in spinal nociceptive processing in chronic inflammation. Pain. 2000;86(3):311-320.

15. Paquet J, Goebel JC, Delaunay C, et al. Cytokines profiling by multiplex analysis in experimental arthritis: which pathophysiological relevance for articular versus systemic mediators? Arthritis Res Ther. 2012;14(2):R60.

16. Infante C, Díaz M, Hernández A, Constandil L, Pelissier T. Expression of nitric oxide synthase isoforms in the dorsal horn of monoarthritic rats: effects of competitive and uncompetitive N-methyl-D-aspartate antagonists. Arthritis Res Ther. 2007;9(3):R53.

17. Butler SH, Godefroy F, Besson JM, Weil-Fugazza J. A limited arthritic model for chronic pain studies in the rat. Pain. 1992;48(1):73-81.

18. Bennett GJ, Xie YK. A peripheral mononeuropathy in rat that produces disorders of pain sensation like those seen in man. Pain. 1988;33(1):87-107.

19. Yang JX, Hua L, LiYQ, et al. Caveolin-1 in the anterior cingulate cortex modulates chronic neuropathic pain via regulation of NMDA receptor 2B subunit. J Neurosci. 2015;35(1):36-52.

20. Dixon WJ. Staircase bioassay: the up-and-down method. Neurosci Biobehav Rev. 1991;15(1):47-50.

21. Livak KJ, Schmittgen TD. Analysis of relative gene expression data using real-time quantitative PCR and the 2(-Delta Delta C(T)) method. Methods. 2001;25(4):402-408.

22. Zhou C, Luo ZD. Electrophysiological characterization of spinal neuron sensitization by elevated calcium channel alpha-2-delta-1 subunit protein. Eur J Pain. 2014;18(5):649-658.

23. Jin XG, Chen SR, Cao XH, Li L, Pan HL. Nitric oxide inhibits nociceptive transmission by differentially regulating glutamate and glycine release to spinal dorsal horn neurons. J Biol Chem. 2011;286(38):33190-33202.

24. Pedersen LM, Jacobsen LM, Mollerup S, Gjerstad J. Spinal cord long-term potentiation (LTP) is associated with increased dorsal horn gene expression of IL-1beta, GDNF and iNOS. Eur J Pain. 2010;14(3):255-260.

25. Ellis A, Bennett DL. Neuroinflammation and the generation of neuropathic pain. Br J Anaesth. 2013;111(1):26-37.

26. Hsieh YL. Effects of ultrasound and diclofenac phonophoresis on inflammatory pain relief: suppression of inducible nitric oxide synthase in arthritic rats. Phys Ther. 2006;86(1):39-49.

27. Hancock CM, Riegger-Krugh C. Modulation of pain in osteoarthritis: the role of nitric oxide. Clin J Pain. 2008;24(4):353-365.

28. Mukherjee P, Cinelli MA, Kang S, Silverman RB. Development of nitric oxide synthase inhibitors for neurodegeneration and neuropathic pain. Chem Soc Rev. 2014;43(19):6814-6838.

29. Lee JS, Zhang Y, Ro JY. Involvement of neuronal, inducible and endothelial nitric oxide synthases in capsaicin-induced muscle hypersensitivity. Eur J Pain. 2009;13(9):924-928.

30. Tanabe M, Nagatani Y, Saitoh K, Takasu K, Ono H. Pharmacological assessments of nitric oxide synthase isoforms and downstream diversity of NO signaling in the maintenance of thermal and mechanical hypersensitivity after peripheral nerve injury in mice. Neuropharmacology. 2009;56(3):702-708.

31. De Alba J, Clayton NM, Collins SD, Colthup P, Chessell I, Knowles RG. GW274150, a novel and highly selective inhibitor of the inducible isoform of nitric oxide synthase (iNOS), shows analgesic effects in rat models of inflammatory and neuropathic pain. Pain. 2006;120(1-2): 170-181.

32. Staunton CA, Barrett-Jolley R, Djouhri L, Thippeswamy T. Inducible nitric oxide synthase inhibition by $1400 \mathrm{~W}$ limits pain hypersensitivity in a neuropathic pain rat model. Exp Physiol. 2018;103(4):535-544.

33. Miletic G, Pankratz MT, Miletic V. Increases in the phosphorylation of cyclic AMP response element binding protein (CREB) and decreases in the content of calcineurin accompany thermal hyperalgesia following chronic constriction injury in rats. Pain. 2002;99(3):493-500.

34. D'Mello R, Dickenson AH. Spinal cord mechanisms of pain. $\mathrm{Br} J$ Anaesth. 2008;101(1):8-16. 


\section{Supplementary materials}
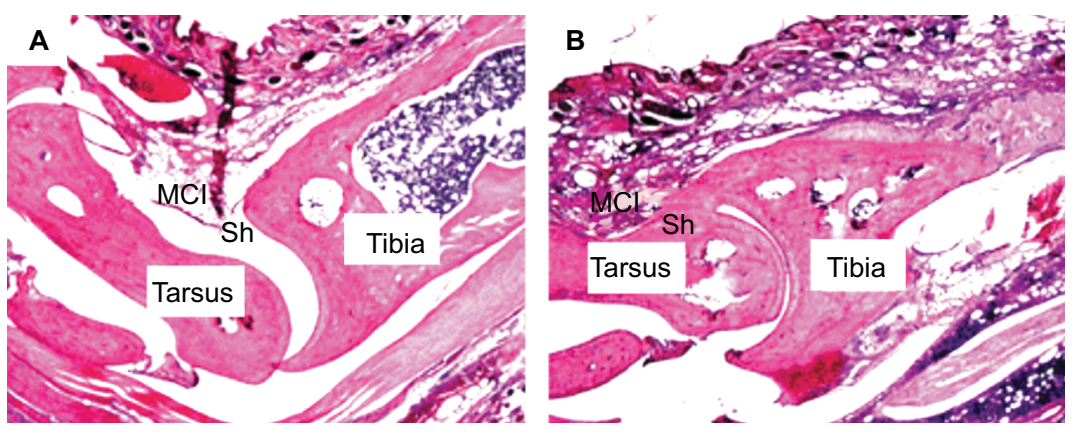

Figure SI CFA-induced histopathological alterations in the tibiotarsal joint.

Notes: Two days after saline or CFA injection in the right tibiotarsal joint, the rats were sacrificed and the right tibiotarsal joints were fixed, sectioned, and subjected to HE staining. A representative histological image of a section of tibiotarsal joint from a sham rat (A) and a CFA rat (B) (40× magnification).

Abbreviations: CFA, complete Freund's adjuvant; HE, hematoxylin-eosin; $\mathrm{MCl}$, mononuclear cell infiltration; Sh, synovial cell lining hyperplasia.

\section{A}

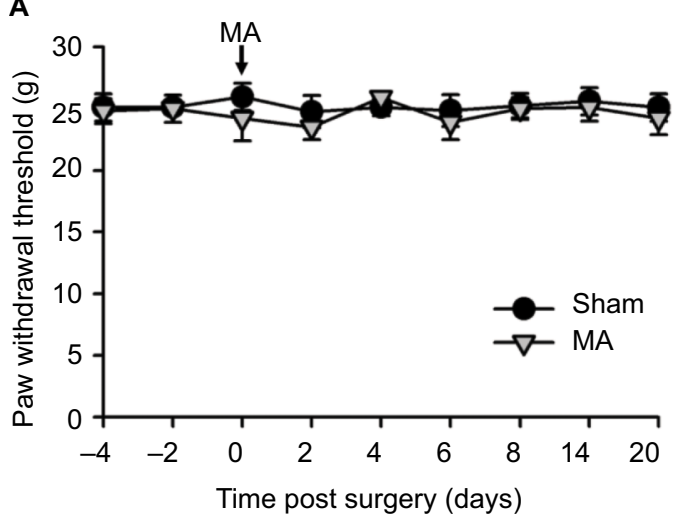

B

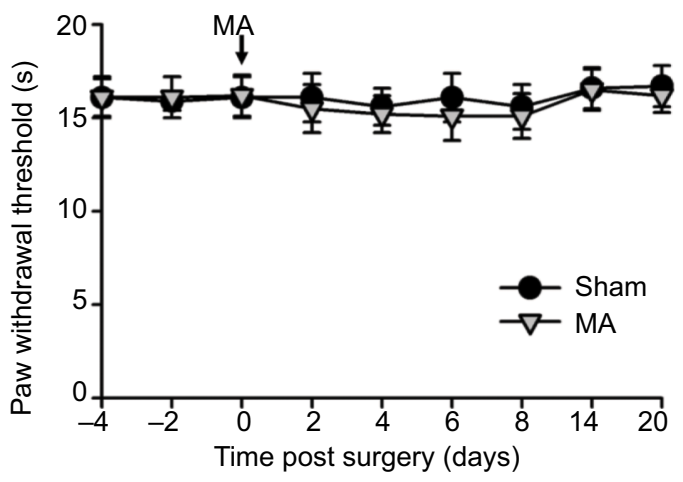

Figure S2 Contralateral MA alone did not induce pain behaviors.

Notes: CFA was injected into the right tibiotarsal joint to induce MA. Saline was injected into the right tibiotarsal joint of the rat in the sham group. Von Frey filament tests and thermal plantar tests were respectively applied to assess mechanical allodynia $(\mathbf{A})$ and heat hyperalgesia $(\mathbf{B})$. Data are shown as mean \pm SEM. $n=6$ in each group. Statistical analysis showed no difference between these two groups.

Abbreviations: CFA, complete Freund's adjuvant; MA, monoarthritis; SEM, standard error of measurement.

Journal of Pain Research

\section{Publish your work in this journal}

The Journal of Pain Research is an international, peer reviewed, open access, online journal that welcomes laboratory and clinical findings in the fields of pain research and the prevention and management of pain. Original research, reviews, symposium reports, hypothesis formation and commentaries are all considered for publication.

\section{Dovepress}

The manuscript management system is completely online and includes a very quick and fair peer-review system, which is all easy to use. Visit http://www.dovepress.com/testimonials.php to read real quotes from published authors. 